\title{
A sepsis gene expression signature in the whole blood.
}

Shahan Mamoor, MS 1

shahanmamoor@gmail.com

Sepsis is a leading cause of mortality ${ }^{1}$. We mined published and public microarray datasets 2,3 to identify differentially expressed genes in the whole blood of patients with sepsis. We observed significant changes in the expression of 78 genes in patients with sepsis and septic shock. Differential expression of these genes was associated with the septic state (independent of infectious agent) and observed in children and adults. The identification of these genes may be useful in the understanding of the biology of sepsis; this gene signature, upon validation, could have clinical relevance as a diagnostic measure.

Keyword: sepsis, septic shock, diagnosis of sepsis, systems biology of sepsis, targeted therapeutics in sepsis and septic shock.

1 OF 18 
Sepsis is a major cause of morbidity and mortality in the United States and worldwide1.

Therapeutic options for patients with sepsis and septic shock are limited. We mined published and public microarray datasets 2,3 to identify genes whose expression was quantitatively most different in the blood of patients with sepsis. We observed significant differential expression of 78 genes in the blood of patients with sepsis, in children and in adults. Each of these genes may be important for the development or progression of sepsis; the gene signature in sum may be relevant as a diagnostic measure.

\section{Methods}

We used datasets GSE1001592 and GSE26440³ for this differential gene expression analysis in conjunction with GEO2R. GSE100159 was generated using Illumina HumanWG-6 v3.0 expression beadchip technology with $n=12$ whole blood from healthy controls and $n=33$ whole blood from patients with sepsis. GSE26440 was generated using Affymetrix Human Genome U133 Plus 2.0 Array technology with $n=32$ whole blood from healthy controls and $n=98$ whole blood from children with septic shock. The Benjamini and Hochberg method of p-value adjustment was used for ranking of differential expression but raw p-values were used for assessment of statistical significance of global differential expression. Log-transformation of data was auto-detected, and the NCBI generated platform annotation was used.

For Gene Ontology (GO) analyses and Reactome pathway analysis, we used the PANTHER online tool ${ }^{4,5}$. For analysis of enriched gene sets, we used the Broad Institute / UC San Diego online tool Molecular Signatures Database (MSigDB)6,7. For computational 
analysis of histone marks at the genomic loci of genes in this gene set (ENCODE), and for identification of predicated transcription factor occupancy at genes in the gene set (ChEA) we used ENRICHr 8,9 .

Statistical tests were performed to evaluate whether expression of each of these genes was significantly different when comparing whole blood from healthy controls and whole blood from patients with sepsis and septic shock using a two-tailed, unpaired t-test with Welch's correction. We used PRISM for all statistical analyses (Version 8.4.0)(455).

\section{Results}

We mined published and public microarray data to identify differentially expressed genes in the whole blood of patients with sepsis in an unbiased fashion and at the systems-level.

A sepsis gene signature in the whole blood.

To identify differentially expressed genes associated with the septic state, we compared whole transcriptome profiles of the whole blood from patients with sepsis with that of whole blood from healthy controls using two independent microarray datasets. We observed significant differential expression of 78 genes in blood from patients with sepsis across both datasets (Table 1). 17 genes in the signature were down-regulated in patient blood (Figure 1).

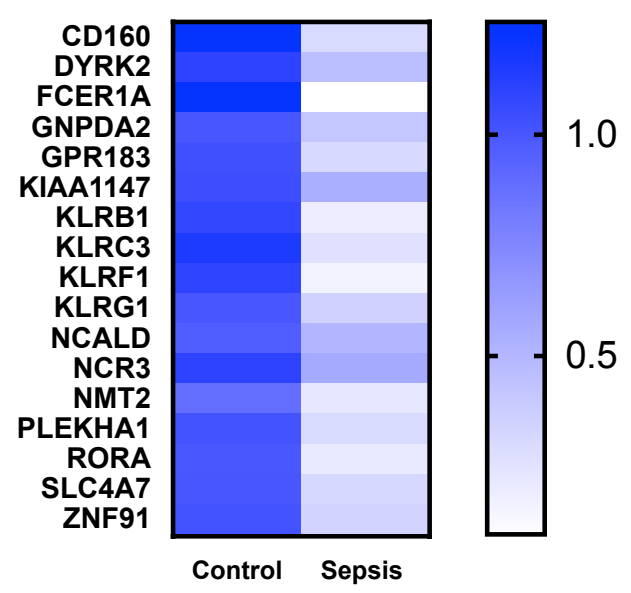

Fig. 1: Genes down-regulated in the whole blood in patients with sepsis and septic shock. Heatmap displaying genes down-regulated in whole blood from patients with sepsis. Data is from GSE26440. 
Notable among the down-regulated genes was a group of killer cell lectin-like receptors KLRB1, KLRC3, KLRF1, and

KLRG1. KLRB1 was down-regulated between $5.45-6.78$ fold (depending on the dataset queried), with $4.24-8.45$ fold downregulation of KLRC3, 6.35 - 6.49 fold down-regulation of 20

KLRF1, and 2.83 - 6.20 fold down-regulation of KLRG1.

Though three Fc receptor genes were part of this signature, only FCER1A was down-regulated.

Fig. 2: Genes up-regulated in the whole blood in patients with sepsis and septic shock.

Heatmap displaying mRNA expression levels of genes upregulated in the 78-member gene signature. The microarray data in this heat map is obtained from dataset GSE26440. CD177 and MMP8 are omitted for scaling purposes.

The expression of 61 of these genes was up-regulated (Figure

2). Genes whose expression was significantly increased in the blood of patients with sepsis included two Fc receptors FCER1G and FCGR1B, innate immune pattern recognition receptors TLR5, NLRC4 and NAIP and lectin-type receptors CLEC4D and CLEC5A, complement receptor CR1, and Bcl proteins BCL2A1 and BCL6. Matrix metalloproteinases MMP8 and MMP9, tyrosine kinase BMX, peptidyl arginine deiminase PADI4, metabolic enzymes HK3 and PFKFB3, and cell adhesion molecules ITGAM and CEACAM were also among genes whose expression was 
significantly higher in whole blood from pediatric and adult patients with sepsis, and more so than a vast majority of the whole blood transcriptome. The magnitude of change in gene expression between septic and normal whole blood for each member of the gene set is listed in Table 1.

We utilized a number of computational tools in an attempt to describe the molecular nature of this sepsis gene signature, derived at in an unbiased manner, in an unbiased fashion. Pathway analyses using the Molecular Signatures Database (MutSigDB) and PANTHER revealed that chief among the pathways enriched based on the presence of genes in this gene set was the process of neutrophil degranulation (Table $2 ; p=1.37 \mathrm{E}-30$ and Table $3 ; p=2.17 \mathrm{E}-22$, respectively for MutSigDB and PANTHER). Two other significantly enriched gene sets were that of the general innate immune response (Table $2 ; p=1.8 \mathrm{E}-26$ ) and a set of genes defining differentiation potential of bipotential myeloid cells (Table $2 ; p=9.51 \mathrm{E}-13$ ). Reactome analysis suggested that immunoregulatory interactions between lymphoid and non-lymphoid cells were enriched as functions of the members of this gene set (Table 3; $p=6.9 \mathrm{E}-07$ ). Gene Ontology (GO) Molecular Function analysis suggested that carbohydrate binding events might be important based on gene set representation (Table $4 ; p=6.07 \mathrm{E}-07$ ).

We used Enrichr to query ENCODE datasets to determine whether any epigenetic marks were enriched for at the genomic loci of these genes. Only one mark, mono-methylation at lysine 4 of histone $\mathrm{H} 3$ (H3K4me1) was enriched at a statistically significant level (Table 5; $p=0.04409)$. In CH12.LX mouse B-cell lymphoma cells, the genomic loci of seven genes in this 
gene set, Siglec5, ITGAM, TLR5, PFKFB3, ALPL, MMP9, and SLC2A3 were marked by H3K4me1. We also used Enrichr to query ChIP enrichment analysis (ChEA) and evaluate transcription factor enrichment among genes of the sepsis whole blood gene set. We identified nine transcription factors and one epigenetic factor whose occupancy at genes of the gene set was significantly enriched for in hematopoietic cell types (Table 6). These included KDM2B $(p=0.0002273)$, SPI1 $(p=0.003506)$, NFYA $(p=0.02399)$, NFYB $(p=0.01088)$, RUNX1 $(p=0.03491), \operatorname{MECOM}(p=0.0198), \operatorname{VDR}(p=0.03965)$, PU.1 $(p=0.02399), \operatorname{CEBPB}(p=0.02399)$, and BCL6 $(p=0.04909)$.

In summary, we identified a whole blood sepsis gene signature using published and public microarray data from pediatric and adult sepsis patients. We further preliminarily characterized the molecular nature of this gene signature using computational tools. Together, this gene signature highlights neutrophil degranulation, cell-cell interactions between lymphoid and non-lymphoid cells and interactions involving carbohydrate-binding, transcriptional changes involving H3K4me1 deposition, and potential involvement of KDM2B, SPI1, NFYA/B, MECOM, and VDR. We also observed enrichment based on overlap with a myeloid differentiation gene set; this could reflect increases in myeloid-derived suppressor cells ${ }^{10,11}$ or emergency myelopoiesis ${ }^{12,13}$. H3K4me1 is a feature of active or poised enhancers ${ }^{14}$ and in mice, endotoxemia results in both $\mathrm{H} 3 \mathrm{~K} 4 \mathrm{me} 1$ deposition at an interferon $\boldsymbol{\gamma}$ (Ifng) enhancer $22 \mathrm{~kb}$ upstream of the transcription start site and in generation of a memory-like natural killer cell subset ${ }^{15}$. It would be of interest to assess $\mathrm{H} 3 \mathrm{~K} 4 \mathrm{me} 1$ deposition and enhancer marking in this 
1

2 3

4

5

6

7

8

9

10

11

12

13

14

15

16

17

18

19

20

21

22

23

24

25

26

27

28

gene signature under physiologic conditions (i.e., human sepsis). The diagnostic value of this gene set, and how it compares with that of existing gene expression-based sepsis diagnostics ${ }^{16-22}$ is unknown.

7 OF 18 


\section{References}

1. Stevenson, E.K., Rubenstein, A.R., Radin, G.T., Wiener, R.S. and Walkey, A.J., 2014. Two decades of mortality trends among patients with severe sepsis: a comparative metaanalysis. Critical care medicine, 42(3), p.625.

2. GSE100159. Nicole Baldwin. BIIR. Dallas, TX, USA. https://www.ncbi.nlm.nih.gov/geo/ query/acc.cgi?acc $=$ GSE 100159 .

3. Wong, H.R., Cvijanovich, N., Lin, R., Allen, G.L., Thomas, N.J., Willson, D.F., Freishtat, R.J., Anas, N., Meyer, K., Checchia, P.A. and Monaco, M., 2009. Identification of pediatric septic shock subclasses based on genome-wide expression profiling. BMC medicine, 7(1), p. 34.

4. Thomas, P.D., Campbell, M.J., Kejariwal, A., Mi, H., Karlak, B., Daverman, R., Diemer, K., Muruganujan, A. and Narechania, A., 2003. PANTHER: a library of protein families and subfamilies indexed by function. Genome research, 13(9), pp.2129-2141.

5. Thomas, P.D., Kejariwal, A., Guo, N., Mi, H., Campbell, M.J., Muruganujan, A. and Lazareva-Ulitsky, B., 2006. Applications for protein sequence-function evolution data: mRNA/protein expression analysis and coding SNP scoring tools. Nucleic acids research, 34(suppl_2), pp.W645-W650.

6. Subramanian, A., Tamayo, P., Mootha, V.K., Mukherjee, S., Ebert, B.L., Gillette, M.A., Paulovich, A., Pomeroy, S.L., Golub, T.R., Lander, E.S. and Mesirov, J.P., 2005. Gene set enrichment analysis: a knowledge-based approach for interpreting genome-wide expression profiles. Proceedings of the National Academy of Sciences, 102(43), pp.15545-15550.

7. Liberzon, A., Birger, C., Thorvaldsdóttir, H., Ghandi, M., Mesirov, J.P. and Tamayo, P., 2015. The molecular signatures database hallmark gene set collection. Cell systems, 1(6), pp. $417-425$

8. Chen, E.Y., Tan, C.M., Kou, Y., Duan, Q., Wang, Z., Meirelles, G.V., Clark, N.R. and Ma'ayan, A., 2013. Enrichr: interactive and collaborative HTML5 gene list enrichment analysis tool. BMC bioinformatics, 14(1), p.128.

9. Kuleshov, M.V., Jones, M.R., Rouillard, A.D., Fernandez, N.F., Duan, Q., Wang, Z., Koplev, S., Jenkins, S.L., Jagodnik, K.M., Lachmann, A. and McDermott, M.G., 2016. Enrichr: a comprehensive gene set enrichment analysis web server 2016 update. Nucleic acids research, 44(W1), pp.W90-W97.

8 OF 18 
10. Delano, M.J., Scumpia, P.O., Weinstein, J.S., Coco, D., Nagaraj, S., Kelly-Scumpia, K.M., O'Malley, K.A., Wynn, J.L., Antonenko, S., Al-Quran, S.Z. and Swan, R., 2007. MyD88dependent expansion of an immature GR-1+CD11b+ population induces T cell suppression and Th2 polarization in sepsis. The Journal of experimental medicine, 204(6), pp.1463-1474.

11. Cuenca, A.G., Delano, M.J., Kelly-Scumpia, K.M., Moreno, C., Scumpia, P.O., LaFace, D.M., Heyworth, P.G., Efron, P.A. and Moldawer, L.L., 2011. A paradoxical role for myeloid-derived suppressor cells in sepsis and trauma. Molecular medicine, 17(3-4), pp. 281-292.

12. Hérault, A., Binnewies, M., Leong, S., Calero-Nieto, F.J., Zhang, S.Y., Kang, Y.A., Wang, X., Pietras, E.M., Chu, S.H., Barry-Holson, K. and Armstrong, S., 2017. Myeloid progenitor cluster formation drives emergency and leukaemic myelopoiesis. Nature, 544(7648), pp. 53-58.

13. Cuenca, A.G., Cuenca, A.L., Gentile, L.F., Efron, P.A., Islam, S., Moldawer, L.L., Kays, D.W. and Larson, S.D., 2015. Delayed emergency myelopoiesis following polymicrobial sepsis in neonates. Innate immunity, 21(4), pp.386-391.

14. Local, A., Huang, H., Albuquerque, C.P., Singh, N., Lee, A.Y., Wang, W., Wang, C., Hsia, J.E., Shiau, A.K., Ge, K. and Corbett, K.D., 2018. Identification of H3K4me1-associated proteins at mammalian enhancers. Nature genetics, 50(1), pp.73-82.

15. Rasid, O., Chevalier, C., Camarasa, T.M.N., Fitting, C., Cavaillon, J.M. and Hamon, M.A., 2019. H3K4me1 supports memory-like NK cells induced by systemic inflammation. Cell Reports, 29(12), pp.3933-3945.

16. Maslove, D.M. and Wong, H.R., 2014. Gene expression profiling in sepsis: timing, tissue, and translational considerations. Trends in molecular medicine, 20(4), pp.204-213.

17. Sweeney, T.E. and Khatri, P., 2017. Benchmarking sepsis gene expression diagnostics using public data. Critical care medicine, 45(1), p.1.

18. Sweeney, T.E., Wong, H.R. and Khatri, P., 2016. Robust classification of bacterial and viral infections via integrated host gene expression diagnostics. Science translational medicine, 8(346), pp.346ra91-346ra91.

19. Sweeney, T.E., Perumal, T.M., Henao, R., Nichols, M., Howrylak, J.A., Choi, A.M., Bermejo-Martin, J.F., Almansa, R., Tamayo, E., Davenport, E.E. and Burnham, K.L., 2018. A community approach to mortality prediction in sepsis via gene expression analysis. Nature communications, 9(1), pp.1-10.

9 OF 18 
20. Sweeney, T.E., Wynn, J.L., Cernada, M., Serna, E., Wong, H.R., Baker, H.V., Vento, M. and Khatri, P., 2018. Validation of the sepsis metascore for diagnosis of neonatal sepsis. Journal of the Pediatric Infectious Diseases Society, 7(2), pp.129-135.

21. Verboom, D.M., Koster-Brouwer, M.E., Varkila, M.R.J., Bonten, M.J.M. and Cremer, O.L., 2019. Profile of the SeptiCyte ${ }^{\mathrm{TM}}$ LAB gene expression assay to diagnose infection in critically ill patients. Expert review of molecular diagnostics, 19(2), pp.95-108.

22. Maslove, D.M., Shapira, T., Tyryshkin, K., Veldhoen, R.A., Marshall, J.C. and Muscedere, J., 2019. Validation of diagnostic gene sets to identify critically ill patients with sepsis. Journal of Critical Care, 49, pp.92-98.

10 OF 18 


\begin{tabular}{|l|r|l|l|r|r|r|}
\hline Gene Set & \#Genes (K) & Description & \# genes (k) & k/K & p-value & FDR q-value \\
\hline $\begin{array}{l}\text { REACTOME_NEUTROPHIL } \\
\text { _DEGRANULATION }\end{array}$ & 479 & Neutrophil degranulation & 26 & 0.0543 & $1.37 \mathrm{E}-30$ & $2.13 \mathrm{E}-26$ \\
\hline $\begin{array}{l}\text { REACTOME_INNATE_ } \\
\text { IMMUNE_SYSTEM }\end{array}$ & 1113 & Innate Immune System & 30 & 0.0270 & $1.8 \mathrm{E}-26$ \\
\hline $\begin{array}{l}\text { BROWN_MYELOID_CELL_ } \\
\text { DEVELOPMENT_UP }\end{array}$ & 161 & $\begin{array}{l}\text { Genes defining differentiation } \\
\text { potential of the bipotential } \\
\text { myeloid cell line FDB. }\end{array}$ & 10 & 0.0621 & $9.51 \mathrm{E}-13$ \\
\hline
\end{tabular}

\section{Table 2: MutSigDB reveals transcriptional features of the sepsis whole blood gene} signature.

Listed is the name of the gene set, number of genes in the gene set $(\mathrm{K})$, description of the gene set, number of genes in the sepsis gene set overlapping with that of the enriched gene set $(\mathrm{k}), \mathrm{k} /$ $\mathrm{K}$, $\mathrm{p}$-value and false discovery rate (FDR) q-value. 


\begin{tabular}{|c|c|c|c|c|c|c|}
\hline Reactome pathways & Total Genes & Genes in Set & Expected & Observed & p-value & FDR \\
\hline Neutrophil degranulation & 478 & 25 & 1.70 & 14.74 & $2.17 \mathrm{E}-22$ & $4.96 \mathrm{E}-19$ \\
\hline $\begin{array}{l}\text { Immunoregulatory interactions } \\
\text { between a Lymphoid and a non- } \\
\text { Lymphoid cell }\end{array}$ & 199 & 8 & 0.71 & 11.33 & $6.9 \mathrm{E}-07$ & $3.15 \mathrm{E}-04$ \\
\hline Innate Immune System & 1105 & 29 & 3.92 & 7.39 & $4.04 \mathrm{E}-18$ & $3.07 \mathrm{E}-15$ \\
\hline
\end{tabular}

\section{Table 3: Reactome pathway analysis indicates involvement of immunoregulatory}

\section{interactions between lymphoid and non-lymphoid cells.}

Listed are the total number of genes in the pathway, number of genes in set overlapping (shared) with genes in pathway, expected enrichment, observed enrichment, $\mathrm{p}$-value and false discovery rate (FDR). 


\begin{tabular}{|l|r|r|r|r|r|r|}
\hline GO Molecular Function & \multicolumn{1}{c}{ Total \# Genes } & \# Genes in Set & Expected & Fold Enrichment & raw P-value & FDR \\
\hline Carbohydrate Binding & 270 & 9 & 0.96 & 9.39 & $6.07 \mathrm{E}-07$ & $2.87 \mathrm{E}-03$ \\
\hline
\end{tabular}

Table 4: Carbohydrate binding is enriched for in the sepsis whole blood gene signature. Listed is the molecular function, the total number of genes in the pathway, number of genes in set overlapping (shared) with genes in pathway, expected enrichment, observed enrichment, raw p-value and false discovery rate (FDR). 


\begin{tabular}{|l|l|l|c|c|}
\hline Epigenetic Mark & Cell Type & P-value & Odds Ratio & Combined score \\
\hline H3K4me1 & CH12.LX & 0.04409 & 1.43 & 4.48 \\
\hline
\end{tabular}

Table 5: Enrichment of the H3K4me1 histone mark in the sepsis whole blood gene signature.

Listed is the type of epigenetic mark enriched, cell type in which this enrichment was experimentally observed, $\mathrm{p}$-value, odds ratio and combined score.

14 OF 18 


\begin{tabular}{|c|c|c|c|}
\hline Name & Tissue / Cell Type & P-value & Odds Ratio \\
\hline KDM2B & K562 & 0.0002273 & 2.41 \\
\hline SPI1 & HL-60 & 0.003506 & 2.43 \\
\hline NFYB & K562 & 0.01088 & 1.90 \\
\hline RUNX & Jurkat & 0.03491 & 2.47 \\
\hline MECOM & Kasumi & 0.01980 & 1.82 \\
\hline VDR & THP-1 & 0.03965 & 2.20 \\
\hline VDR & THP-1 & 0.02991 & 1.97 \\
\hline PU.1 & Macrophages & 0.02399 & 1.77 \\
\hline СЕВРВ & Macrophages & 0.02399 & 1.77 \\
\hline PU.1 & Macrophages & 0.02399 & 1.77 \\
\hline NFYA & K562 & 0.02399 & 1.77 \\
\hline BCL6 & CML-JURL-MK1 & 0.04909 & 1.65 \\
\hline
\end{tabular}

Table 6: Computationally identified transcription and epigenetic factor occupancy in genes of the sepsis whole blood gene signature.

Listed is the nuclear factor, the tissue or cell type or cell line in which this occupancy was experimentally observed, $\mathrm{p}$-value and odds ratio. 


\begin{tabular}{|c|c|c|c|c|}
\hline & Mean_FC & SDFC & $\begin{array}{l}\text { Mean FC } \\
\text { (II) }\end{array}$ & SD FC (W) \\
\hline ADGRG3 & 6.536 & 3.545 & 5.838 & 2.653 \\
\hline $\mathrm{ADM}$ & 4.519 & 2.668 & 3.902 & 1.145 \\
\hline AGFG1 & 4.529 & 2.648 & 4.996 & 2.593 \\
\hline ALPL & 6.469 & 4.519 & 4.391 & 1.596 \\
\hline ANKRD22 & 19.867 & 20.561 & 49.171 & 41.828 \\
\hline ANXA3 & 16.803 & 10.865 & 12.279 & 7.459 \\
\hline BASP1 & 3.122 & 1.197 & 2.383 & 0.572 \\
\hline BCL2A1 & 6.733 & 4.607 & 9.876 & 7.662 \\
\hline BCL6 & 4.576 & 2.361 & 3.151 & 0.840 \\
\hline BMX & 9.353 & 7.125 & 27.539 & 18.309 \\
\hline BST1 & 3.801 & 2.104 & 3.903 & 1.395 \\
\hline CA4 & 10.476 & 8.155 & 9.033 & 4.666 \\
\hline CD160 & 0.244 & 0.257 & 0.111 & 0.176 \\
\hline CD177 & 42.375 & 34.797 & 99.139 & 71.494 \\
\hline CEACAM1 & 11.395 & 8.521 & 13.799 & 9.117 \\
\hline CKAP4 & 6.163 & 3.490 & 5.612 & 2.767 \\
\hline CLEC4D & 9.638 & 7.831 & 12.389 & 9.949 \\
\hline CLEC5A & 14.978 & 13.950 & 12.264 & 9.692 \\
\hline CR1L/CR1 & 2.764 & 1.139 & 9.882 & 4.745 \\
\hline DYRK2 & 0.418 & 0.207 & 0.221 & 0.148 \\
\hline DYSF & 5.987 & 3.079 & 5.317 & 2.288 \\
\hline FCER1A & 0.077 & 0.124 & 0.088 & 0.189 \\
\hline FCER1G & 3.411 & 1.354 & 3.746 & 1.635 \\
\hline FCGR1B & 7.711 & 6.340 & 9.909 & 6.888 \\
\hline GADD45A & 5.958 & 4.448 & 6.056 & 3.331 \\
\hline GNPDA2 & 0.423 & 0.156 & 0.260 & 0.147 \\
\hline GRB10 & 7.544 & 5.618 & 8.516 & 5.282 \\
\hline GYG1 & 7.643 & 4.316 & 7.835 & 3.760 \\
\hline GPR84 & 13.745 & 11.510 & 84.803 & 85.834 \\
\hline
\end{tabular}

Table 1: Differentially expressed genes in whole blood from patients with sepsis.

$\underline{\text { FC: }}$ Fold change. SD: Standard deviation. Data here are from GSE26440 and GSE100159 (II).

16 OF 18 
1

2

3

4

5

6

7

8

9

10

11

12

13

14

15

16

17

18

19

20

21

22

23

24

25

26

27

\begin{tabular}{|c|c|c|c|c|}
\hline & $\underline{\text { Mean FC }}$ & $\underline{\text { SD FC }}$ & Mean FC (II) & $\underline{\text { SD FC (II) }}$ \\
\hline GPR183 & 0.306 & 0.217 & 0.157 & 0.140 \\
\hline $\begin{array}{l}\text { HIST2H2AA4/ } \\
\text { HIST2H2AA3 }\end{array}$ & 3.198 & 1.310 & 4.196 & 1.377 \\
\hline HK3 & 6.046 & 3.544 & 6.401 & 4.077 \\
\hline HP & 22.666 & 19.290 & 60.513 & 45.952 \\
\hline IRAK3 & 7.022 & 4.157 & 9.469 & 4.576 \\
\hline ITGAM & 3.493 & 1.761 & 3.608 & 1.283 \\
\hline KIAA1147 & 0.530 & 0.170 & 0.245 & 0.180 \\
\hline KLRB1 & 0.184 & 0.148 & 0.148 & 0.126 \\
\hline KLRC3 & 0.236 & 0.249 & 0.118 & 0.163 \\
\hline KLRF1 & 0.154 & 0.236 & 0.158 & 0.132 \\
\hline KLRG1 & 0.353 & 0.169 & 0.161 & 0.162 \\
\hline KIF1B & 4.283 & 2.130 & 3.961 & 1.649 \\
\hline LILRB3 & 2.522 & 1.074 & 2.642 & 0.694 \\
\hline LIMK2 & 2.860 & 1.336 & 7.725 & 4.555 \\
\hline LIN7A & 3.828 & 2.364 & 4.598 & 1.428 \\
\hline LMNB1 & 3.067 & 1.486 & 4.009 & 1.860 \\
\hline LRG1 & 6.809 & 4.578 & 5.709 & 2.692 \\
\hline MAPK14 & 4.477 & 2.834 & 7.424 & 4.046 \\
\hline MCEMP1 & 18.630 & 9.757 & 18.006 & 8.969 \\
\hline METTL9 & 2.453 & 0.867 & 3.380 & 1.178 \\
\hline MMP8 & 155.915 & 159.218 & 388.845 & 394.136 \\
\hline MMP9 & 17.714 & 14.178 & 15.845 & 8.543 \\
\hline NAIP & 7.544 & 6.772 & 9.084 & 5.965 \\
\hline NCALD & 0.527 & 0.188 & 0.143 & 0.144 \\
\hline NCR3 & 0.521 & 0.160 & 0.162 & 0.128 \\
\hline NLRC4 & 5.838 & 3.908 & 7.408 & 3.625 \\
\hline NMT2 & 0.257 & 0.253 & 0.140 & 0.122 \\
\hline OSCAR & 4.133 & 2.383 & 3.687 & 1.484 \\
\hline PADI4 & 5.864 & 3.606 & 3.759 & 1.373 \\
\hline
\end{tabular}

Table 1: Differentially expressed genes in whole blood from patients with sepsis.

FC: Fold change. SD: Standard deviation. Data here are from GSE26440 and GSE100159 (II). 
2

3

4

5

6

7

8

9

10

11

12

13

14

15

16

17

18

19

20

21

22

23

24

25

26

27

\begin{tabular}{|c|c|c|c|c|}
\hline & $\underline{\text { Mean FC }}$ & $\underline{\text { SD FC }}$ & Mean FC (II) & $\underline{\text { SD FC (II) }}$ \\
\hline PGD & 5.514 & 2.885 & 3.604 & 1.516 \\
\hline PGLYRP1 & 6.919 & 6.155 & 6.715 & 3.527 \\
\hline PFKFB3 & 7.802 & 4.898 & 8.632 & 4.621 \\
\hline PLEKHA1 & 0.298 & 0.186 & 0.179 & 0.121 \\
\hline RORA & 0.214 & 0.204 & 0.180 & 0.115 \\
\hline SIGLEC5 & 3.871 & 2.102 & 4.359 & 1.681 \\
\hline SIPA1L2 & 3.648 & 2.089 & 5.209 & 2.323 \\
\hline SIRPA & 3.502 & 1.927 & 2.536 & 0.783 \\
\hline SLC2A14/SLC2A3 & 5.563 & 2.707 & 7.866 & 3.949 \\
\hline SLC2A3 & 5.081 & 2.898 & 2.666 & 0.616 \\
\hline SLC4A7 & 0.319 & 0.186 & 0.329 & 0.158 \\
\hline STOM & 4.131 & 1.977 & 4.324 & 1.900 \\
\hline SULT1B1 & 3.567 & 2.245 & 3.118 & 1.184 \\
\hline SYN2 & 2.132 & 0.831 & 11.616 & 7.992 \\
\hline TDRD9 & 9.619 & 9.034 & 11.616 & 7.992 \\
\hline TLR5 & 5.768 & 3.233 & 5.870 & 2.379 \\
\hline USB1 & 2.345 & 0.766 & 3.122 & 1.065 \\
\hline VNN1 & 13.555 & 10.145 & 15.420 & 9.601 \\
\hline ZNF91 & 0.334 & 0.199 & 0.304 & 0.132 \\
\hline ZNF438 & 2.925 & 1.386 & 3.599 & 1.441 \\
\hline
\end{tabular}

Table 1: The most differentially expressed genes in whole blood from patients with sepsis (3/3). FC: Fold change. SD: Standard deviation. Data here are from GSE26440 and GSE100159 (II). 